\title{
Risk perception among Brazilian individuals with high risk for colorectal cancer and colonoscopy
}

\author{
Erika M Santos ${ }^{*}{ }^{\dagger}$, Maria TC Lourenço and Benedito M Rossi
}

\begin{abstract}
Background: Risk perception is considered a motivating factor for adopting preventive behaviors. This study aimed to verify the demographic characteristics and cancer family history that are predictors of risk perception and to verify if risk perception is a predictor of colonoscopy adherence.

Methods: Individuals with a family colorectal cancer history as indicated by a proband with cancer were interviewed by telephone. They responded to a questionnaire covering demographic characteristics, colonoscopy history and four questions on risk perception. Tests of multiple linear regression and logistic regression were used to identify associations between dependent and independent variables.

Results: The 117 participants belonged to 62 families and had a mean age of 45.2 years. The majority of these individuals were female (74.4\%) and from families who met the Amsterdam Criteria (54.7\%). The average risk perception was $47.6 \%$, with a median of $50 \%$. The average population perception of individual risk was $55.4 \%$, with a median of 50\%. Variables associated with a higher risk perception were age, gender, religion, school level, income, and death of a family member. The variable predicting colonoscopy was receiving medical information regarding risk (odds ratio OR 8.40).
\end{abstract}

Conclusions: We found that family cancer history characteristics (number of relatives with cancer, risk classification) are associated with adequate risk perception. Risk perception does not predict colonoscopy in this sample. The only variable that predicted colonoscopy was receiving medical information recommending screening.

\section{Background}

According to the Brazilian National Cancer Institute (INCA) [1], colorectal cancer (CRC) is the third most common cancer in Brazil, with an estimated 28,000 new cases expected to be diagnosed in 2010 [1]. The main risk factors for CRC development are family history, age, and dietary habits [2]. In a meta-analysis, Butterworth et al. [3] identified 59 studies published from 1958 to 2004 on CRC risk with a grouped relative risk for CRC in individuals with family history of 2.14 ( $95 \%$ confidence interval (CI) 1.98-2.32). Hereditary conditions can be associated with an increase in risk of up to $100 \%[2]$.

Mortality associated with CRC can be reduced by early detection. A systematic review found that the use of the fecal occult blood test (FOBT) reduces the risk of CRC death by $16 \%$ [4]. A 15-year clinical study found

\footnotetext{
* Correspondence: erikammsantos@gmail.com

† Contributed equally

Graduation Program, Antonio Prudente Foundation, Sao Paulo, Brazil
}

that regular colonoscopies in high-risk individuals have reduced the incidence and mortality of CRC [5].

In 2003, INCA published recommendations for CRC screening with FOBT for individuals with a general population risk (50 years or older with no other additional risk factors), and colonoscopy for positive FOBT tests. This recommendation also indicates that individuals with a family history of CRC should be evaluated by a health professional to recommend testing for CRC early detection [6].

Although CRC is ideally suited for early detection measures, the willingness of individuals to adhere to screening recommendations is low. According to the 2000 National Health Interview Survey, only $45 \%$ of men and $41 \%$ of women aged 50 years or more reported completion of FOBT in the previous year or colorectal endoscopy in the last 10 years [7].

Because the participation is considered low, it is necessary to understand why individuals do not adhere to screening recommendations. Risk perception is
Ciomed Central

() 2011 Santos et al; licensee BioMed Central Ltd. This is an Open Access article distributed under the terms of the Creative Commons Attribution License (http://creativecommons.org/licenses/by/2.0), which permits unrestricted use, distribution, and reproduction in any medium, provided the original work is properly cited. 
among the factors cited in the literature as predictors of having a colonoscopy [8-10].

Collins et al. [8] evaluated 114 individuals tested for a predisposition to Lynch Syndrome and found that the only variable associated with colonoscopy before the test was the perception of risk (odds ratio (OR) 1.03; 95\%CI 1.00-1.05). Risk perception, or susceptibility, refers to the subjective risk of an individual to develop a certain condition or disease [11-13]. Depending on the evaluation of the threat, it is considered a motivating factor for the adoption of preventive or protective behaviors $[14,15]$. It is thought that bringing risk perception and actual risk values closer enables the individual to adopt health behaviors appropriate to the level of risk $[14,16]$; however, this idea has yielded conflicting results in the literature [16].

In addition to studying the association between risk perception and health behavior, it is important to evaluate which aspects are associated with overestimation or underestimation of risk. Several characteristics have been associated with changes in risk perception, including gender $[17,18]$, age $[18]$, family history $[18-20]$, and educational level [19].

It should be noted that the majority of studies refer to North American and European samples. Because of the scarcity of studies in Latin-American populations, this study aims (1) to verify which demographic characteristics and cancer family history are predictors of risk perception, and (2) to verify if risk perception is a predictor of colonoscopy adherence.

\section{Methods}

This descriptive and cross-sectional study was performed at a teaching hospital in Sao Paulo, Brazil and was approved by the Institutional Review Board. The inclusion criteria were individuals older than 18 years of age without a personal history of cancer who were firstdegree relatives of a patient with CRC who fulfilled the Bethesda criteria [21] or whose family met the Amsterdam criteria [22,23].

To identify the research participants, patients with CRC were selected through the institutional hereditary CRC registry that was established in 1992. Patients received a letter requesting authorization for the researcher to enter into contact with their first-degree relatives. Each consenting participant signed two copies of an informed consent form and provided a list of their relatives.

A telephone call was made to the potential research volunteers to verify participation in the research. After the first contact, they received an invitation letter and two copies of an informed consent form, and a telephone interview was then scheduled. The telephone interview followed a script that included a questionnaire with demographic characteristics and information pertaining to colonoscopy history. Risk perception measures were based on Clarke et al. [24], as follows:

- numeric scale of population risk perception: Individuals must choose between $0 \%$ (meaning no risk of the individual to develop CRC) and 100\% (absolute certainty that the individual will develop CRC) about the chance of an individual from the general population to develop CRC;

- numeric scale of personal risk perception: Individuals must choose between $0 \%$ (meaning no risk of the individual's having CRC) and 100\% (absolute certainty that the individual will have CRC) about the individual's own risk of developing CRC;

- verbal scale of personal risk perception: Individuals must indicate their own risk of developing CRC, using a verbal scale (low risk, medium risk, high risk);

- comparative verbal scale of personal risk perception: Individuals must indicate their own risk of having CRC compared with the general population risk, using a verbal scale (lower risk, same risk, higher risk).

The comparative verbal scale of personal risk perception was used to classify risk perception as adequate or inadequate. For individuals that estimated their risk perception as higher or very higher in the comparative scale they were classified as adequate. Individuals that estimated their risk perception as very low, low or average were considered with inadequate risk perception.

Colonoscopy compliance was measured considering risk classification. For individuals that belong into families that fulfilled the Amsterdam Criteria, colonoscopy compliance was considered adequate if they reported a colonoscopy in the past two years. For individuals whose family member fulfilled the Bethesda Criteria, colonoscopy compliance was considered adequate if they reported a colonoscopy in the past five years.

The t-test or ANOVA was used to identify the association between demographic characteristics and family history and risk perception variables [25]; the chi-square test was used for analyzing independent variables and categorical, dependent variables (risk perception or colonoscopy).

After selection of the independent variables with a statistical significance cut-off of 0.20 , a multiple regression was done. A multiple linear regression was used for the continuous dependent variables and multiple logistic regression for the categorical, dependent variables. Tests with $\mathrm{p}$ values less than 0.05 were considered statistically significant [25]. A multiple logistic regression was also used to identify factors that predicted colonoscopy in 
this sample. SSPS version 15 was used for statistical analysis.

\section{Results \\ Sample characteristics}

A total of 152 patients were identified. Of these, 62 returned the informed consent and provided a list of names and contact information of family members (there were 70 non-respondents, 9 refusals, and 11 cases for which the address was not valid). The 62 patients indicated a total of 180 relatives. Of this total, 39 were not located (a letter was sent and there were three attempts made to reach them by phone), 16 refused, and 8 were excluded because they were identified as second-degree relatives after the pedigree analysis. Thus, the sample consisted of 117 participants.

Table 1 shows the sample characteristics. The participant mean age was 45.2 years (range 18-70). Most respondents were less than 50 years old (62.4\%); most

\section{Table 1 Sample characteristics}

\begin{tabular}{|c|c|c|}
\hline Sample characteristics & $\mathrm{N}$ & $\%$ \\
\hline \multicolumn{3}{|l|}{ Age } \\
\hline Up to 50 years & 73 & 62.4 \\
\hline 50 years or more & 44 & 37.6 \\
\hline \multicolumn{3}{|l|}{ Gender } \\
\hline Female & 97 & 74.4 \\
\hline Male & 30 & 25.6 \\
\hline \multicolumn{3}{|l|}{ Education level } \\
\hline Elementary & 22 & 18.8 \\
\hline High-school & 46 & 39.3 \\
\hline College & 49 & 41.9 \\
\hline \multicolumn{3}{|l|}{ Family income(monthly)* } \\
\hline Up to US\$1,098 & 31 & 26.5 \\
\hline From US\$1,098 to US\$2,746 & 27 & 23.1 \\
\hline More than US\$2,747 & 33 & 28.2 \\
\hline \multicolumn{3}{|l|}{ Religion } \\
\hline Catholic/other & 100 & 85.5 \\
\hline Evangelic/Protestant & 9 & 7.7 \\
\hline \multicolumn{3}{|l|}{ Risk classification } \\
\hline Bethesda criteria & 53 & 45.3 \\
\hline Amsterdam criteria & 64 & 54.7 \\
\hline \multicolumn{3}{|c|}{ Death of a family member from colorectal cancer } \\
\hline No & 24 & 20.5 \\
\hline Yes & 93 & 79.5 \\
\hline \multicolumn{3}{|c|}{ Received information regarding colorectal risk } \\
\hline No & 12 & 10.3 \\
\hline Yes & 105 & 89.7 \\
\hline \multicolumn{3}{|l|}{ Colonoscopy compliance } \\
\hline No or Inadequate & 65 & 55.6 \\
\hline Yes & 52 & 44.4 \\
\hline Total & 117 & 100.0 \\
\hline
\end{tabular}

* 26 individuals did not respond. were female (74.4\%); $41.9 \%$ had a college education; and $88.5 \%$ were Catholic. Most came from families who met the Amsterdam criteria (54.7\%), and $79.5 \%$ reported that at least one family member had died from cancer. When asked whether they had received information about CRC risk, $89.7 \%$ answered yes, and $82.1 \%$ reported knowledge regarding colonoscopy. From the total, 44.4\% colonoscopy compliance was considered adequate.

\section{Risk perception}

The mean of population risk perception, according to numeric scale, was $47.6 \%$ with a median of $50 \%$. The average perception of individual risk was $55.4 \%$ with a median of $55 \%$.

Twenty-three (19.7\%) individuals did not address risk perception with numerical scales. Factors that predicted response to risk perception evaluated by numerical scales were individuals 50 years or less (OR 3.30; 95\%CI 1.26-8.69) and those with a college degree (OR 3.15; 95\% CI 1.06-9.42).

Of the 115 individuals who responded to the question about risk perception with a verbal scale, $58.3 \%$ reported a high risk of developing CRC and $41.7 \%$ reported a low or medium risk. According to the comparative verbal scale, $69.0 \%$ reported higher risk, and $31 \%$ reported lower risk or the same (one respondent did not answer this question).

Table 2 presents the multiple linear regression results for the population risk perception according to the numerical scale and Table 3 for personal risk perception according to the numerical scale. The variables kept in the model were responsible for $18.8 \%$ of the variance in perception of population risk. The standardized regression coefficients indicate that female gender, elementary school education level, and family income lower than US $\$ 1,098 /$ month were associated with an overestimation of population risk according to the numerical scale.

Regarding personal risk perception according to the numerical scale, variables kept in the model were responsible for $28.5 \%$ of the variance. Variables associated with greater perception of personal risk were family history (fulfillment of the Amsterdam criteria) and religion (Catholic/other).

Table 4 presents the results of multiple logistic regression for individual risk according to the verbal scale. The verbal scale model accounted for $32.4 \%$ of the variance. Catholic/other religion, age ( $\leq 50$ years), female gender, and family income ( $\geq$ US $\$ 2,747 /$ month) were associated with a higher risk perception according to the verbal scale.

Table 5 presents the results of multiple logistic regression for individual risk according to the comparative verbal scale. The comparative verbal scale model accounted for $28.1 \%$ of the variance. Death of a family member and female gender were associated with higher 
Table 2 Multiple linear regression model for population risk perception according to the numeric scale

\begin{tabular}{lllcc}
\hline Adjusted R2 & Statistical significance of the model & Model variables & Standardized Coefficients $(\boldsymbol{\beta})$ & $\mathbf{p}$ \\
\hline 0.188 & $<0.001$ & Education: elementary & 0.249 & 0.017 \\
& & Gender: female & 0.216 & 0.025 \\
& Income: less than US\$1,098 & 0.206 & 0.048 \\
\hline
\end{tabular}

risk perception according to the comparative verbal scale.

The risk perception was considered inadequate in 32 (27.4\%) of the participants and adequate in 85 (72.6\%) of the participants. The only variable that predicted adequate risk perception was the number of family members with cancer; having three or more family members with $\mathrm{CRC}$ resulted in more accurate risk perception (OR 4.72 p 0.023).

\section{Risk perception and colonoscopy}

Risk perception was associated with colonoscopy only when measured with the comparative scale. Of the 36 individuals who reported having a lower or the same risk as individuals from the general population, 16 (45\%) had undergone a colonoscopy, while $54(67.9 \%)$ of the 80 individuals who reported higher risk had undergone the procedure $(\mathrm{p}=0.015)$.

Based on the multivariate analysis, risk perception did not predict colonoscopy. The only variable that predicted colonoscopy was receiving medical information recommending screening (OR 8.40; 95\%IC 1.55-48.71).

\section{Discussion}

In the search for the motives that lead some individuals to have an exam and others to choose not to, an increasing number of studies since the 1950s have evaluated health behaviors related to cancer prevention. This study is one of the first to evaluate CRC-related health behaviors in Brazil.

The assessment of health behaviors is important in different cultures, as are differences in risk perception. Kim et al. [26] evaluated 1160 women with perceived risk of cancer of the breast, cervix, and colon and noted that Latinas had a perception of higher risk compared to Whites, African-Americans, and Asians.

Although there is no screening program for CRC in Brazil, some institutions have established programs for high-risk individuals. Understanding the beliefs of high- risk individuals may contribute to establishing educational and psychosocial interventions that take into account not only the technical and scientific knowledge of health professionals but also the beliefs and attitudes of lay people.

The low response frequency of solicited patients to indicate their relatives $(40.8 \%)$ should be noted. One of the possible explanations for this outcome is that patients did not want their relatives to be contacted in order to preserve the family. Koehly et al. [27], evaluating the communication pattern of five Lynch Syndrome families, verified that communication among members of the family depends on the psychosocial characteristics of their relationships [27].

Regarding the number of individuals who responded to questions on risk perception, a lesser number of individuals responded to questions about risk perception using a numeric scale as compared to questions using a verbal scale. This fact may highlight the difficulties individuals experience in estimating using a numeric scale. Although this study did not assess numeracy, these results point to the need to do so; according to Keller and Siegrist [28], different formats in the communication of risk perception produce different effects on risk perception, and numeracy mediates these differences. Kelly et al. [29] evaluated the numeric comprehension of women and their relation to risk perception and verified that younger women with more education presented greater numeric comprehension.

Individuals in this study overestimated the CRC population risk. An overestimation may reflect a lack of numeric ability, an influence of experiences with cancer, or a coping mechanism.

According to Price et al. [30], to estimate individual risk, the individual uses personal characteristics and behavior. To estimate population risk, the individual identifies the frequency of the event in the population, and the belief of individual risk does not depend on the

Table 3 Multiple linear regression model for personal risk perception according to the numeric scale

\begin{tabular}{|c|c|c|c|c|}
\hline Adjusted R2 & Statistical significance of the model & Model variables & Standardized coefficients $(\beta)$ & $\mathrm{p}$ \\
\hline \multirow[t]{5}{*}{0.285} & $<0.001$ & Amsterdam criteria: yes & 0.332 & 0.001 \\
\hline & & Religion: Catholic & 0.261 & 0.007 \\
\hline & & Gender: female & 0.179 & 0.063 \\
\hline & & Age: up to 50 years & 0.149 & 0.124 \\
\hline & & Received information: yes & 0.142 & 0.145 \\
\hline
\end{tabular}


Table 4 Multiple logistic regression for personal risk perception according to the verbal scale

\begin{tabular}{|c|c|c|c|}
\hline Variable & $\mathrm{OR}^{*}$ & $95 \% \mathrm{Cl}$ & $\mathbf{p}$ \\
\hline \multicolumn{4}{|l|}{ Religion } \\
\hline Protestant & 1 & & \\
\hline Catholic/other & 5.3 & $2.1-21.3$ & 0.010 \\
\hline \multicolumn{4}{|l|}{ Age } \\
\hline$>50$ years & 1 & & \\
\hline$\leq 50$ years & 3.8 & $1.4-10.2$ & 0.006 \\
\hline \multicolumn{4}{|l|}{ Gender } \\
\hline Male & 1 & & \\
\hline Female & 3.4 & $1.2-9.9$ & 0.023 \\
\hline \multicolumn{4}{|l|}{ Family income } \\
\hline$<$ US\$2,746 & 1 & & \\
\hline$\geq$ US\$2,747 & 3.3 & $1.1-9.8$ & 0.032 \\
\hline \multicolumn{4}{|c|}{ Relatives with cancer who died } \\
\hline 0 & 1 & & \\
\hline 1 or more & 3.0 & $0.9-9.4$ & 0.058 \\
\hline
\end{tabular}

* OR: Ratio of chances refers to high individual risk according to the verbal scale.

perception of frequency of the event in the population. Individuals who are considered high risk, based on their judgment of personal characteristics, may judge that an event is relatively frequent [30].

The median of population risk and numeric scale individual risk in this study was $50 \%$. Klein and Stefanek [31] highlighted the difficulties individuals experience in establishing strategies to cope with numeric information and pointed out that " $50 \%$ " may not be used in numeric form but as an expression of doubt about the occurrence of an event, especially to estimate their own risk

Table 5 Multiple logistic regression for individual risk perception with the verbal and comparative verbal scales

\begin{tabular}{|c|c|c|c|}
\hline Variable & $\mathrm{OR}^{*}$ & $95 \% \mathrm{Cl}$ & $\mathrm{p}$ \\
\hline \multicolumn{4}{|l|}{ Relatives with cancer who died } \\
\hline 0 & 1 & & \\
\hline 1 or more & 5.7 & $1.7-19.0$ & 0.005 \\
\hline \multicolumn{4}{|l|}{ Gender } \\
\hline Male & 1 & & \\
\hline Female & 3.3 & $1.2-9.1$ & 0.017 \\
\hline \multicolumn{4}{|l|}{ Family income } \\
\hline$<$ US\$2,746 & 1 & & \\
\hline$\geq$ US\$2,747 & 3.4 & $0.8-13.6$ & 0.085 \\
\hline \multicolumn{4}{|l|}{ Education level } \\
\hline Elementary/high-school & 1 & & \\
\hline College & 1.9 & $0.6-5.9$ & 0.255 \\
\hline \multicolumn{4}{|l|}{ Amsterdam criteria } \\
\hline No & 1 & & \\
\hline Yes & 1.6 & $0.6-4.3$ & 0.326 \\
\hline
\end{tabular}

* OR: Ratio of chances of individuals refers to high individual risk according to the verbal scale and higher individual risk according to the comparative verbal scale. in an uncertain situation [31]. Risk perception is considered an essentially cognitive evaluation, and it is believed that the perception of risk also includes emotional reactions, especially with a verbal scale $[32,33]$. According to Bottorf et al. [34], the conversion of numeric information to a verbal scale (low, medium, or high risk) is a mechanism to simplify complex information in a way that facilitates the decision process.

Women reported a perception of greater population risk than men. Health perception in women is influenced by context, not only personal context but also that of others around them. Because women traditionally assume caring roles, contact with the experiences of relatives is greater and as such leads to more reaction to events than men may have $[35,36]$.

Lower income was associated with overestimation of the risk population. This may reflect the socioeconomic status, which may indicate that individuals with less lower income have more difficulty in interpreting numerical information. This assumption may also explain why people with higher incomes reported greater perception of risk in accordance with the verbal scale.

Despite the fact that mutation analysis was not performed in this sample, since all participants had at least one family member with CRC diagnosed before 45 years old, we considered those individuals with a higher risk of CRC when compared with general population. However, $27.4 \%$ of this sample indicated their risk as lower or the same than general population. The only variable that predicted adequate risk perception was the number of family members with cancer which may suggest that family experiences with the disease can modulate risk perception.

Risk perception did not predict colonoscopy. However, medical advice was a factor in the performance of colonoscopy, as other studies have found [37-39]. Therefore, health care professionals have an important role informing individuals regarding the need to be submitted to a colonoscopy.

This study has some limitations. It is a cross-sectional study and therefore did not assess changes in risk perception over time. Moreover, the selection of participants occurred from the indication of the proband. This procedure was adopted because of ethical aspects associated with the fact that we had no direct initial contact with family members, only with patients. However, this study is one of the first done in Brazil to evaluate health behaviors in individuals at high risk for CRC.

\section{Conclusions}

We found that family cancer history characteristics (number of relatives with cancer, objective risk classification) are associated with adequate risk perception. Risk perception does not predict colonoscopy in this 
sample. The only variable that predicted colonoscopy was receiving medical information recommending screening.

\section{List of Abbreviations}

Cl: Confidence interval; CRC: Colorectal cancer; FOBT: fecal occult blood test; INCA: Brazilian National Cancer Institute; OR: Odds Ratio

\section{Authors' contributions}

EMMS: conceived of the study, interviewed participants and performed the statistical analysis. MTCL: participated in the study design and helped to draft the manuscript. BMR: participated in the study design and helped to draft the manuscript. All authors read and approved the final manuscript.

\section{Competing interests}

The authors declare that they have no competing interests.

Received: 25 May 2010 Accepted: 28 July 2011 Published: 28 July 2011

\section{References}

1. Brazil.INCA: [Estimativa 2010: Incidência de Câncer no Brasil]. Rio de Janeiro, INCA; 2009.

2. Duarte-Franco E, Franco EL: [Epidemiologia e fatores de risco em cancer colorretal[.Edited by: Rossi BM et al. Sao Paulo, Lemar/Tecmedd; 2004:3-22, [Cancer de colon, reto e ânus].

3. Butterworth AS, Higgins JP, Pharoah P: Relative and absolute risk of colorectal cancer for individuals with a family history: a meta-analysis. Eur J Cancer 2006, 42:216-227.

4. Hewitson P, Glasziou P, Irwing L, et al: Screening for colorectal cancer using the faecal occult blood test, Hemoccult. Oxford, The Cochrane Library; 2007.

5. Jarvinen $\mathrm{HJ}$, Aarnio $\mathrm{M}$, Mustonen $\mathrm{H}$, et al: Screening reduces colorectal cancer rate in families with hereditary nonpolyposis colorectal cancer. Gastroenterology 1995, 108:1405-1411.

6. Brazil INCA: [Falando sobre o câncer de intestino. Orientações úteis ao usuário sobre fatores de risco e proteção] Rio de Janeiro, INCA; 2003.

7. Seeff $L C$, Nadel MR, Klabunde $C N$, et al: Patterns and predictors of colorectal cancer test use in the adult U.S. population. Cancer 2004, 100:2093-2103

8. Collins V, Meiser B, Gaff C, et al: Screening and preventive behaviors one year after predictive genetic testing for hereditary nonpolyposis colorectal carcinoma. Cancer 2005, 104:273-281.

9. Codori AM, Petersen GM, Miglioretti DL, et al: Health beliefs and endoscopic screening for colorectal cancer potential for cancer prevention. Prev Med 2001, 33:128-136.

10. Palmer RC, Emmons KM, Fletcher RH, et al: Familial risk and colorectal cancer screening health beliefs and attitudes in an insured population. Prev Med 2007, 45:336-341.

11. Janz NK, Champion VL, Strecher VJ: The Health Belief Model. In Health Behavior and Health Education: Theory, Research and Practice. Edited by: Glanz K, Rimer BK, Lewis FM. San Francisco, Jossey-Bass; 2002:45-66.

12. Glanz K, Lewis FM, Rimer BK, et al: Theory at a Glance: a Guide for Health Promotion Practice. Washington, National Institute of Health; 1997.

13. Rosenstock IM: Historical origins of the health belief model. Health Educ Monograph 1974, 2:328-335.

14. Vernon SW: Risk perception and risk communication for cancer screening behaviors: a review. J Natl Cancer Inst Monogr 1999, 19:101-119.

15. Weinstein ND: What does it mean to understand a risk? Evaluating risk comprehension. J Natl Cancer Inst Monogr 1999, 19:15-20.

16. Leventhal H, Kelly K, Leventhal EA: Population risk, actual risk, perceived risk, and cancer control: a discussion. J Natl Cancer Inst Monogr 1999, 25:81-85.

17. Green PM, Kelly BA: Colorectal cancer knowledge, perceptions, and behaviors in African Americans. Cancer Nurs 2004, 27:206-215.

18. Robb KA, Miles A, Wardle J: Demographic and psychosocial factors associated with perceived risk for colorectal cancer. Cancer Epidemiol Biomarkers Prev 2004, 13:366-372.
19. Stark JR, Bertone-Johnson ER, Costanza ME, et al: Factors associated with colorectal cancer risk perception: the role of polyps and family history. Health Educ Res 2006, 21:740-749.

20. Friedman LC, Webb JA, Richards CS, et al: Psychological and behavioral factors associated with colorectal cancer screening among Ashkenazim. Prev Med 1999, 29:119-125.

21. Umar A, Boland CR, Terdiman JP, et al: Revised Bethesda Guidelines for hereditary nonpolyposis colorectal cancer (Lynch syndrome) and microsatellite instability. J Natl Cancer Inst 2004, 96:261-268.

22. Vasen HF, Mecklin JP, Khan PM, et al: The International Collaborative Group on Hereditary Non-Polyposis Colorectal Cancer (ICG-HNPCC). Dis Colon Rectum 2001, 34:424-425

23. Vasen HF, Watson P, Mecklin JP, et al: New clinical criteria for hereditary nonpolyposis colorectal cancer (HNPCC, Lynch syndrome) proposed by the International Collaborative group on HNPCC. Gastroenterology 1999, 116:1453-1456.

24. Clarke VA, Lovegrove H, Williams A, et al: Unrealistic optimism and the Health Belief Model. J Behav Med 2000, 23:367-376.

25. Vieira S: Bioestatistica: Topicos Avancados. Rio de Janeiro, Campus; 2003.

26. Kim SE, Pérez-Stable EJ, Wong S, et al: Association between cancer risk perception and screening behavior among diverse women. Arch Intern Med 2008, 168:728-734.

27. Koehly LM, Peterson SK, Watts BG, et al: A social network analysis of communication about hereditary nonpolyposis colorectal cancer genetic testing and family functioning. Cancer Epidemiol Biomarkers Prev 2003, 12:304-313.

28. Keller $\mathrm{C}$, Siegrist M: Effect of risk communication formats on risk perception depending on numeracy. Med Decis Making 2009, 29:483-490

29. Kelly KM, Graves KD, Harper FW, et al: Assessing perceptions of cancer risk: does mode of assessment or numeracy matter? Cancer Detect Prev 2007, 31:465-473.

30. Price PC, Pentecost HC, Voth RD: Perceived event frequency and the optimistic bias: evidence for a two-process model of personal risk judgments. J Exp Soc Psychol 2002, 38:242-252.

31. Klein WM, Stefanek ME: Cancer risk elicitation and communication: lessons from the psychology of risk perception. CA Cancer J Clin 2007, 57:147-167.

32. Levy AG, Shea J, Williams SV, et al: Measuring perceptions of breast cancer risk. Cancer Epidemiol Biomarkers Prev 2006, 15:1893-1898.

33. Fagerlin A, Zikmund-Fisher BJ, Ubel PA: "If I'm better than average, then I'm ok?": Comparative information influences beliefs about risk and benefits. Patient Educ Couns 2007, 69:140-144.

34. Bottorff JL, Ratner PA, Johnson JL, et al: Communicating cancer risk information: the challenges of uncertainty. Patient Educ Couns 1998, 33:67-81.

35. Benyamini Y, Leventhal EA, Leventhal H: Gender differences in processing information for making self-assessments of health. Psychosom Med 2000, 62:354-364.

36. Koopmans GT, Lamers LM: Gender and health care utilization: the role of mental distress and help-seeking propensity. Soc Sci Med 2007, 64:1216-1230.

37. Honda K, Neugut Al: Associations between perceived cancer risk and established risk factors in a national community sample. Cancer Detect Prev 2004, 28:1-7.

38. Klabunde CN, Schenck AP, Davis WW: Barriers to colorectal cancer screening among Medicare consumers. Am J Prev Med 2006, 30:313-319.

39. Guessous I, Dash C, Lapin P, et al: Colorectal cancer screening barriers and facilitators in older persons. Prev Med 2010, 50:3-10.

doi:10.1186/1897-4287-9-4

Cite this article as: Santos et al:: Risk perception among Brazilian individuals with high risk for colorectal cancer and colonoscopy. Hereditary Cancer in Clinical Practice 2011 9:4. 